\title{
Assessing Adverse Events in Madeira Primary Health Care
}

\author{
Marta Dora Ornelas ${ }^{1(\varpi)}\left(\right.$ D and Paulo Sousa ${ }^{2,3}$ \\ ${ }^{1}$ SESARAM, E.P.E, Rua das Hortas 67, 9050-024 Funchal, Portugal \\ phdmarta@gmail.com \\ 2 National School of Public Health, Av. Padre Cruz, 1600-560 Lisbon, Portugal \\ ${ }^{3}$ CISP - Centro de Investigação em Saúde Pública, ENSP - Universidade Nova \\ de Lisboa, Lisbon, Portugal
}

\begin{abstract}
In last three decades, several epidemiological studies have been developed in order to assess the magnitude, nature and type of adverse events (AEs). Most of these studies focus on hospital settings, where the activities are more standardised, but simultaneously more complex and involving higher risks.

However, in the last years, there is a growing movement and strong evidence that point out the importance of studying other healthcare contexts, such as primary care and long-term care. In Portugal, studies on primary care setting are scarce and still in the early stages.

In this article, the authors describe the AEs assessment in Portuguese Primary Health Care (PHC) units in Madeira Island/Portugal. This study was quantitative, cross-sectional, observational and analytical, with probability sampling. We quantify and analyse the AEs registered by healthcare providers using the APEAS-PT formulary.

A link to the APEAS-PT form was sent to 520 healthcare professionals (111 specialist in Family Medicine, 27 medical students, 382 nurses) who worked in 32 PHC centres. These professionals identified and analysed 85 AEs and 42 incidents, which corresponds to a prevalence of 3.9 AEs per 10,000 visits, with a 95\% confidence interval (CI) between 3.7 and 4 AE. Most of the AEs were preventable (96\%). The most frequent causal factors of AEs were associated with medication $(69 \%)$, health care provided to users $(54 \%)$, communication $(41 \%)$ and diagnosis $(22 \%)$.

This analysis of AEs in Madeira island PHC contributed to reinforce patient safety culture and to better understand quaternary prevention.
\end{abstract}

Keywords: Adverse events $\cdot$ Patient safety $\cdot$ Primary Health Care

\section{Introduction}

The quality of medical care has been a topic of social concern since Mesopotamia (18th century BC), where the Code of Hammurabi stated the legal responsibility of medical practice in the event of malpractice - 'eye for an eye, tooth for a tooth'. In ancient Greece (460-351 BC), Hippocrates of Kos, the father of medicine, formulated the 
principle of non-maleficence, Primum Non Nocere: 'To patients, has by habit two things: help or at least not produce damage' [1].

With the publication of 'To Err is Human: Building a Safer Health System' [2] and several other studies such as the Harvard Medical Practice Study, [3] the issue of patient safety (PS) and its more media-interesting component, the adverse events (AEs), were launched into the limelight and subjected to public opinion.

The Institute of Medicine in 2000 defined an AE as 'any negative occurrence occurred beyond the will and as a result of treatment, but not the disease that has resulted, causing some damage, from a simple disturbance of clinical work (incident) to permanent harm or even death' [2].

In the last decades, numerous efforts have been made to understand the nature and magnitude of AEs and to propose appropriate solutions. Most of these studies focus on hospital settings, where the activities are more standardised, but simultaneously more complex and involving higher risks [4].

In Portugal, Fragata estimates that the number of deaths caused by medical errorsis 1,300-2,900 annually [5]. More recently, Sousa et al., through the analysis of a random sample of 4250 medical records (representative of 180000 hospital admissions) for 2013, found an incidence rate of $12.5 \%$ AEs, of which around 39.9\% were considered preventable [6]. In Portuguese Primary Health Care (PHC) these studies are particularly scarce.

Portuguese family practitioners (FP) act as gatekeepers for hospital care and are responsible for family planning, follow-up of child development, surveillance of lowrisk pregnancies, chronic disease management and care of the elderly. Most general practices have 5-10 FP working in a group, and most doctors care for 1,500-1,900 patients [7].

Given the particularities of PHC, it is clear that patient safety (PS) principles and methods in the hospital context cannot simply be transposed into this level of care [8].

The APEAS study [9] was the first epidemiological study to assess AEs in PHC. This study took place in 2008, involved 452 healthcare professionals and determined the prevalence of AEs in Spanish PHC as $18.63 \%$ (with a prevalence of $11.18 \%$ o for AEs and 7.45\% for incidents); $70 \%$ of these events were considered preventable.

Makeham et al. in 2008 estimated an incidence of AEs in PHC of 0.0004-24\% and, similarly to what happens in hospitals, $45-76 \%$ were found to be preventable [10].

In an area such as medicine, it is true that the vulnerability of those who are ill is associated with the inevitability of the occurrence of AEs by those who must exercise care in susceptible environments and systems; however, it is no less true that the adoption of a safety culture (with recognition, quantification and evaluation of this public health problem in a transparent and non-punitive way) by the national health service in general and by the PHC in particular, is a proven weapon to make AEs less likely and to minimise their consequences, where they may but inevitably occur [11].

In 2016, we conduct a pilot study in Madeira Island [12] that aimed to assessed Patient Safety Culture (PSC) in PHC. This study constituted an important contribution to (i) identify strengths and areas for improvement; (ii) create PS awareness among PHC professionals; and (iii) contribute to the first strategic objective of the Portuguese Plan for Patient Safety ("increase the safety culture of the internal environment"). Based on the results of this pilot study on PSC, the authors began the quantification and 
analysis of AEs in Portuguese PHC with the application of APEAS formulary [9] (which was translated, adapted and validated for the Portuguese population). Besides addressing the issue of the prevalence of AEs, in this study we also sought to understand the possible etiology, contributing / causal factors and the consequences of these events.

In this chapter we describe the results obtained in that pioneering study that aims to estimate the incidence, nature and impact of AEs in Madeira PHC. This was an exploratory study that also intended to examine the feasibility of applying to Portuguese PHC the methodology previously used in other countries.

\section{Materials and Methods}

This study was quantitative, cross-sectional, observational and analytical, with probability sampling.

In order to obtain more reliable and accurate results, the process of translation, adaptation and application of APEAS formulary was performed in five steps: original version translation, conceptual equivalence evaluation, acceptability and viability assessment, content validity and psychometric properties assessment. The internal consistencies of the subscales were examined using Cronbach's alpha.

From November 2016 to April 2017, a link to the APEAS-PT form was sent to 520 healthcare professionals (111 general practice physicians, 27 general practice medical students, 382 nurses) who worked in 32 PHC centres.

Health professionals during a consultation with a patient and in presence or suspected occurrence of an Adverse Event (EA) or a security incident (current or previous), they completed the APEAS form. To ensure that the forms were as complete as possible, health professionals used the "interview" with the patient (direct/ face collection) and the consultation of the respective clinical process (indirect).

The target population included caregivers (e.g. medical specialists, training physicians, nurses) and the rest of the healthcare team (technical assistants, operational assistants, nutritionists, social workers and psychologists) who performed functions in centres that make up the PHC of RAM. Health professionals who worked in more than one service were asked to restrict their responses to the place where they had the greatest workload.

Exclusion criteria to integrate the sample was defined as RAM health centres with fewer than 3 caregivers (medical specialists, training physicians and nurses) or fewer than 5 participants, and participants with less than 1 month of experience at the place where we applied the questionnaire. Due to its logistics and location, PHC in Porto Santo island did not participate in this research work.

Completed questionnaires with more than 5 missing answers or the same answer to all questions in at least 1 section (with questions positively and negatively listed, sections C, D, E and F) or with 'sequential responses' (e.g. C1 strongly disagree, C2 Disagree, C3 Neither agree nor disagree, C4 agree) also were excluded from the data analysis.

Data were collected and statistically worked using - Microsoft Excel 2010 and IBM Statistical Package for Social Sciences - IBM SPSS version 23. The statistical treatment 
of the data was based on the use of descriptive methods and methods of statistical inference.

The International Classification of PS [13] was used, and AEs were considered as the unexpected and undesirable events that happen to a patient caused by provided health care.

\section{Results}

We obtained 331 returned links/entries, of which 152 (46\%) were considered valid.

The healthcare providers identified and analyzed 85 AEs and 42 incidents, which corresponds to a prevalence of 3.9 AEs per 10,000 visits, with a $95 \%$ confidence interval (CI) of $3.7-4$ AEs. The prevalence of AEs reported by physicians was of three events per 10,000 visits $(\mathrm{CI}-2.3-3.1)$ and in nurses this prevalence was of six AEs per 10,000 visits with a CI of 5.2-6. Most of the AEs were preventable (96\%).

In Table 1 we present the notifications submitted by patient safety incident. The percentage of non-response to this question was $13.8 \%$; $9.9 \%$ of the AEs caused permanent damage and $4.6 \%$ resulted in death.

Table 1. Notifications submitted by typology of patient safety incident

\begin{tabular}{l|c|l}
\hline Security patient safety incident & Number & Percentage \\
\hline No security incident occurred but was close to happening & 4 & 2.6 \\
\hline An incident occurred and was detected before it affected the patient & 4 & 2.6 \\
\hline $\begin{array}{l}\text { An incident occurred that affected the patient but do not cause } \\
\text { damage }\end{array}$ & 38 & 25.0 \\
\hline AE occurred, and the patient was damaged for a period of time & 53 & 34.9 \\
\hline AE occurred, and the patient was in a critical situation & 10 & 6.6 \\
\hline AE occurred, and the patient was permanently damaged & 15 & 9.9 \\
\hline AE occurred which resulted in death of the patient & 7 & 4.6 \\
\hline No answer & 21 & 13.8 \\
\hline Total & 152 & 100 \\
\hline
\end{tabular}

Diabetes and hypertension was the intrinsic risk factors more frequently present in patients with AEs in the two APEAS studies (in Spain and Portugal).

The PHC users who suffered incidents were, on average, 54.5 years old, $60 \%$ were women and $72 \%$ had at least one risk factor or disease. The events were more frequent in patients with hypertension, diabetes, heart failure and renal failure ( $p$-value $<0.05)$. Anxiety, stress, depression, need to repeat procedures or consultations, and worse evolutionary course of the underlying disease were more frequent in patients with AEs.

In our study, women were the ones most affected $(60 \%)$. This result, being in line with what happened in Spain [9]. contradicts the data verified in the AEs study performed in Portuguese hospitals [6]. 
The most frequent causal factors of AEs selected by healthcare providers from regional PHC centres were associated with medication $(69 \%)$, health care provided to users (54\%), communication (41\%) and diagnosis (22\%) - Table 2.

Table 2. Causal factors of AEs most frequently selected by healthcare providers in APEAS

\begin{tabular}{l|l|l}
\hline Causal factors of AEs & $\begin{array}{l}\text { APEAS }- \text { PT } \\
(\%)\end{array}$ & $\begin{array}{l}\text { Spanish APEAS } \\
(\%)\end{array}$ \\
\hline Adverse drug reaction & 34.1 & 26 \\
\hline Inappropriate care for the patient/users & 30.6 & 13.7 \\
\hline Inappropriate care to the patient at warning signs & 22.4 & 8.9 \\
\hline Improperly applied technique & 18.8 & 10.4 \\
\hline $\begin{array}{l}\text { Problems of communication between doctor and } \\
\text { patient }\end{array}$ & 17.6 & 15 \\
\hline Problems of communication between doctors & 11.8 & 3.6 \\
\hline Delay in diagnosis & 11.8 & 7.5 \\
\hline Insufficient monitorisation or follow up & 9.4 & 1.9 \\
\hline Long waiting list/delay in patient observation & 8.2 & 5.3 \\
\hline Wrong medication management & 7.1 & 3.9 \\
\hline
\end{tabular}

As a consequence of AEs identified, we observed that in $61.2 \%$ of the cases, more observation and monitoring were required in Madeira PHC (Spain 33.1\%), 37.6\% had to undergo additional treatments (in Spain $17.1 \%$ ) and $21.2 \%$ of the cases $(7.5 \%$ in Spain) required complementary diagnostic tests (performed at health centers). Hospital resources were also used following these unexpected events (AEs) in about $45 \%$ of the reports. In Spain, there were more AEs requiring hospital consultation (24.9\% versus $18.8 \%$ in Madeira) and fewer events causing hospitalization and / or life support treatments $(5.8 \%$ versus $25.9 \%$ in Madeira Island), which is in agreement with the severity of the events reported in Madeira.

\section{Discussion}

In Spain, the prevalence of AEs was 8 per 10,000 consultations [9] (twice as high as in Madeira); this high rate, however, may also represent a greater and better PSC in Spanish PHC centres, with more notifications and awareness for this problem.

In the Spanish APEAS study, [9] 452 professionals from 48 PHC centres made 2,059 notifications in 2 weeks. In our study, the 152 valid forms which correspond to 152 notifications result in a $30 \%$ participation rate in the 6 months of data collection. This rate is clearly low but slightly higher than that observed in the 2005 study carried out by a Portuguese network of sentinel doctors, where despite the participating professionals being highly motivated for the notifications, only $28 \%$ reported one or more AEs in a year, with a total of 208 notifications [11]. The National System for Notification of Incidents and Adverse Events (NOTIFIQ@) system, which includes notifications from all levels of care, has a total of notifications that is far from reflecting 
national reality $(1,675$ notifications made by health professionals and 159 notifications from patients or their relatives) from 2013 to 2015 [14].

According to the literature, in the studies that use questionnaires as a tool for colecting data on safety incidents in PHC, the participation / response rate varies between $29 \%$ [15] and $76 \%$ [16], being that than $50 \%$ were observed in only one study [16]. Unfortunately underreporting is a well known problem and an unavoidable limitation of voluntary reporting systems by health professionals. Due to the fact that the data analysed refer to voluntary notification / registration of AEs by health professionals, the participation rate was low (30\%), which is in agreement with that described in the literature [9].

The difficulty of professionals in recognizing AEs [17, 18], problems with the definition of concepts [19, 20], fear of reprisals or the formulation of value judgments by the employers [21, 22], concerns about the anonymity and confidentiality of the notifications, the lack of time to report and the suspicion or uncertainty of the possible effect that notification may have [23] are priority areas of work when we want high participation rates of health professionals.

Communication errors in PHC vary with results of 4.5\%, [24] 15\% [9, 25, 26] and 72\%. [27] In our study, communication problems between doctors and patients was $17.6 \%$. The main communication problems in PHC of Madeira island occurred between physicians and patients and among physicians (11.8\%). Communication error between PHC physicians in our study was problematic in a percentage of notifications, almost four times higher than that observed in the Spanish APEAS study [9]. The same was verified in the communication between doctors and nurses $(3.5 \%$ vs. $0.8 \%$ in Spain).

\section{Conclusions}

As the first level of contact for individuals, family and the community, PHC brings health care as close as possible to where people live and work, with more personal and longer-term care that are the key points for the design of effective preventive strategies. Its high rate of users, makes the occurrence of AEs in PHC a real public health issue. The perception of occurrence of these events by health professionals varies according to the safety culture present in their workplaces.

The APEAS-PT focus is on primary, secondary, tertiary and even quaternary prevention. By investigating the risk factors (intrinsic and extrinsic) of the patients and the factors that contributed to the occurrence of AE, the methodology inherent to the APEAS study supports primary prevention; on the other hand, by promoting the early diagnosis of the damage, its correction and minimization of its impact on the patient contributes to both secondary and tertiary prevention; Last but not least, it focuses on quaternary prevention, identifying safe and acceptable practices for patients, and avoiding the overuse of complementary diagnostic exams, unnecessary treatments and therapeutic procedures [3].

The results of this study can be seen as a first step to contribute to reinforce patient safety culture and to better understand quaternary prevention. 


\section{References}

1. Neto, J.A., Sirimarco, M.T., Figueiredo, N.S., Barbosa, T.N., Silveira, T.G.: Erro Médico - A perspectiva de estudantes de Medicina e Direito [The perspective of medical and law students]. Rev. Bras. Educ. Med. 35(1), 5-12 (2011)

2. Kohn, L.T., Corrigan, J.M., Donaldson, M.S.: To Err is Human: Building a Safer Health System. National Academy Press, Washington, DC (2000)

3. Brennan, T.A., Leape, L.L., Laird, N.M., et al.: Incidence of adverse events and negligence in hospitalized patients: results of the Harvard Medical Practice study I. N. Engl. J. Med. 324 (6), 370-376 (1991). https://doi.org/10.1056/NEJM199102073240604

4. Mendes, C.M., Barroso, F.F.: Promover uma cultura de segurança em cuidados de saúde primários [Promote a culture of safety in Primary Health Care]. Rev Por Saúde Pública. 32 (2), 197-205 (2014)

5. Fragata, J., Martins, L.: O Erro em Medicina [The Mistake in Medicine]. Almedina, Coimbra (2009)

6. Sousa, P., Uva, A.S., Serranheira, F., Uva, M.S., Nunes, C.: Patient and hospital characteristics that influence incidence of adverse events in acute public hospitals in Portugal: a retorspective cohort study. Int. J. Qual. Health Care, 1-6 (2018). https://doi.org/ 10.1093/intqhe/mzx190

7. Granja, M., Ponte, C., Cavadas, L.: What keeps family physicians busy in Portugal? A multicentre observacional study of work other than direct patient contacts. BMJ Open 4, 111 (2014). https://doi.org/10.1136/bmjopen-2014005026

8. Wetzels, R., Wolters, R., van Weel, C., Wensing, M.: Harm caused by adverse events in primary care: a clinical observational study. J. Eval. Clin. Pract. 15(2), 323-327 (2009). https://doi.org/10.1111/j.1365-2753.2008.01005.x

9. Agencia de Calidad del Sistema Nacional de Salud. Estudio APEAS: Estudio Sobre La Seguridad de Los Pacientes en Atención Primaria de Salud. [APEAS Study: Study on the Safety of Patients in Primary Health Care]. Madrid (2008)

10. Makeham, M., Dovey, S., Runciman, W., Larizgoitia, I.: Methods and measures used inprimary care patient safety research: results of a literature review. World Heal Organ (2008)

11. Ribas, M.J.: Eventos adversos em cuidados de saúde primários: Promover uma cultura de segurança [Adverse events in Primary Health Care: promoting a culture of safety]. Rev Port Clin Geral. 26(Dossier: Erro Médico), 585-589 (2010)

12. Ornelas, M.D., Pais, D., Sousa, P.: Patient safety culture in Portuguese primary healthcare. Qual. Prim. Care. 24(5), 214-218 (2016)

13. Departamento da Qualidade na Saúde - DGS. Estrutura Concetual Da Classificação Internacional Sobre Segurança Do Doente [Conceptual Framework of the International Classification on Patient Safety]. Lisboa (2011)

14. Departamento da Qualidade na Saúde - DGS. Tipologia de Incidentes Notificados Top 3 Profissional Top 3 - Cidadão [Typology of Notified Incidents Top 3 - Professional Top 3 Citizen] (2015)

15. Elder, N.C., Pallerla, H., Regan, S.: What do family physicians consider an error? A comparison of definitions and physician perception. BMC Fam. Pract. 7, 73 (2006)

16. McKay, J., Bowie, P., Murray, L., Lough, M.: Attitudes to the identification and reporting of significant events in general practice. Clin. Gov. An. Int. J. 9(2), 96-100 (2004)

17. Tamuz, M., Thomas, E.J., Franchois, K.E.: Defining and classifying medical error: lessons for patient safety reporting systems. Qual. Saf. Heal. Care 13(1), 13-21 (2004) 
18. Hobgood, C., Xie, J., Weiner, B., Hooker, J.: Error identification, disclosure, and reporting: practice patterns of three emergency medicine provider types. Acad. Emerg. Med. 11(2), 196-199 (2004)

19. Jeffe, D.B., Dunagan, W.C., Garbutt, J., et al.: Using focus groups to understand physicians' and nurses' perspectives on error reporting in hospitals. J. Qual. Saf. [Internet] 30(9), 471-9 (2004). http://www.ncbi.nlm.nih.gov/entrez/query.fcgi?cmd=Retrieve\&db=Pub-Med\& dopt=Citation\&list_uids $=15469124$

20. Dovey, S.M., Phillips, R.L.: What should we report to medical error reporting systems? Qual. Saf. Heal. Care 13(5), 322-323 (2004)

21. Taylor, J.A., Brownstein, D., Christakis, D.A., et al.: Use of incident reports by physicians and nurses to document medical errors in pediatric patients. Pediatrics 114(3), 729-735 (2004)

22. Karsh, B., Hamilton, K., Beasley, J.W., Holden, R.J.: Toward a theoretical approach to medical error reporting system research and design. Appl. Ergon. 37(3), 283-295 (2006)

23. Elder, N.C., Graham, D., Brandt, E., Hickner, J.: Barriers and motivators for making error reports from family medicine offices: a report from the American Academy of Family Physicians National Research Network (AAFP NRN). J. Am. Board Fam. Med. [Internet], 20(2), 115-23 (2007). http://www.ncbi.nlm.nih.gov/pubmed/17341747

24. Elder, N.C.: The identification of medical errors by family physicians during outpatient visits. Ann Fam Med. 2(2), 125-129 (2004). https://doi.org/10.1370/afm.16

25. Rosser, W., Dovey, S., Bordman, R., White, D., Crighton, E., Drummond, N.: Medical errors in primary care: results of an international study of family practice. Can. Fam. Physician 51, 386-387 (2005)

26. Makeham, M.B., Dovey, S.M., County, M., Kidd, M.R.: An international taxonomy for errors in general practice: A pilot study. Med. J. Aust. 177(2), 68-72 (2002)

27. Westfall, J.M., Fernald, D.H., Staton, E.W., Vanvorst, R., West, D., Pace, W.D.: Applied strategies for improving patient safety: a comprehensive process to improve care in rural and frontier communities. J. Rural. Heal. 20(4), 355-362 (2004). https://doi.org/10.1111/j.17480361.2004.tb00049.x 